\title{
Platoon-Based Driving Protocol for Multimedia Transmission Over VANET
}

\author{
Wellington Lobato Junior ${ }^{1}$, Denis Rosário ${ }^{1}$ (Orientador), \\ Leandro A. Villas ${ }^{2}$ (Co-orientador) \\ ${ }^{1}$ Programa de Pós-Graduação em Engenharia Elétrica (PPGEE) \\ Universidade Federal do Pará (UFPA) \\ Belém - Pará - Brasil \\ ${ }^{2}$ Instituto de Computação (UNICAMP), Campinas - São Paulo - Brasil \\ \{wellingotnl, denis\}@ufpa.br, leandro@ic.unicamp.br
}

\begin{abstract}
Multimedia content will be one of the main services of vehicular networks (VANETs) and is becoming a reality in the environment of smart cities, ranging from safety traffic warnings to live entertainment, in addition to being fundamental to the development of autonomous vehicles. However, VANETs have a dynamic network topology with the short contact time, which leads to communication flaws and delays, increasing packet loss, and decreasing the Quality of Experience ( $Q o E$ ) of transmitted videos. To cope with this, neighbor vehicles moving in the same direction and wishing to cooperate should form a platoon, where platoon members act as a relay node to forward video packets. This master's dissertation introduces a Game Theory approach for Platoonbased driving (GT4P - Game Theory for Platoon) for video dissemination services in urban and highway VANET scenarios. GT4P encourages cooperation between neighbor vehicles by offering a reward (money or coupon) for vehicles participating in the platoon. Simulation results confirm the efficiency of GT4P for ensuring video transmissions with high QoE support compared to existing platoon-based driving protocols.
\end{abstract}

Resumo. Conteúdo multimídia será um dos principais serviços das redes veiculares (VANETS) e está se tornando uma realidade no ambiente de cidades inteligentes, que vão desde avisos de tráfego de segurança até entretenimento ao vivo, além de fundamental para o desenvolvimento de veículos autônomos. No entanto, as VANETs possuem uma topologia de rede dinâmica com pouco tempo de contato entre os veículos, o que leva a falhas de comunicação, aumentando a perda de pacotes e diminuindo a Qualidade de Experiência (QoE) dos vídeos transmitidos. Para lidar com isso, os veículos vizinhos que se movem na mesma direção e desejam cooperar podem formar um pelotão, onde os membros do pelotão atuam como um nó de retransmissão para encaminhar pacotes de vídeo. Esta dissertação de mestrado introduz uma abordagem de teoria de jogos para a condução baseada em pelotão (GT4P - Game Theory for Platoon) para serviços de disseminação de vídeo em cenários urbanos e rodoviários de VANET. O GT4P incentiva a cooperação entre os veículos vizinhos oferecendo recompensa (dinheiro ou cupom) para os veículos que participam do pelotão. Os resultados da simulação confirmam a eficiência do GT4P para garantir transmissões de vídeo com suporte a QoE em comparação aos protocolos baseados em pelotão existentes. 


\section{Introdução e Justificativa}

A disseminação de vídeo já é um importante impulsionador do mercado, e onde não são compartilhados apenas vídeos pessoais, mas também vídeos publicitários ou de entretenimento em redes de veiculares (VANETs) [Ulin 2019]. Contudo, a disseminação de vídeo em VANET requer suporte de Qualidade de Experiência (QoE), levando em consideração a satisfação do usuário ao assistir o conteúdo, onde os usuários esperam assistir a vídeo em tempo-real sem interrupções, fantasmas, bloqueio, pixelização e congelamento de quadros, independentemente das alterações nas condições da rede e das características da VANET [Lopez-Martin et al. 2018]. Essa é uma tarefa desafiadora devido às frequentes desconexões causadas devido as características intrínsecas das VANETs, tal como os veículos geralmente se movem em alta velocidade com duração limitada na comunicação Veículo para Veículo (V2V) [Husain et al. 2019]. Por exemplo, [Uppoor and Fiore 2012] analisou a mobilidade de Colônia-Alemanha e concluiu que os contatos entre pares de veículos são extremamente curtos, ou seja, entre 1 e 15 segundos. Portanto, como mitigar a influência do curto tempo de contato nas comunicações V2V para evitar falhas na comunicação, atrasos e perda de pacotes durante as transmissões de vídeo em VANET ainda é uma tarefa desafiadora [Liu et al. 2016].

Veículos vizinhos que se movam na mesma direção com interesses em comum podem cooperar para estabelecer um pelotão, onde o líder do pelotão define a velocidade, direção e distância entre os membros do pelotão, dando instruções sobre quando acelerar ou frear [Li et al. 2018]. A mobilidade baseada em pelotão provê a disseminação de dados ou vídeo mais eficientes entre os membros do pelotão, pois evita falhas de comunicação na comunicação V2V. Apesar dos benefícios de dirigir com base em pelotões do ponto de vista do sistema, os usuários têm um comportamento egoísta, levando à entrada de veículos por pouco tempo em pelotões, causando perdas de pacotes e desconexões [Ahmad et al. 2019]. A decisão de ingressar no pelotão depende do motorista, considerando os benefícios enquanto faz uma rota possivelmente mais longa para chegar ao local de destino. O comportamento cooperativo pode ser reforçado por meio de recompensa (dinheiro ou cupom) para incentivar os veículos participantes do pelotão, uma vez que os usuários tendem a ser egoístas. Desta forma, a teoria dos jogos é uma ferramenta eficaz para modelar um mecanismo de incentivo, bem como para que nós inativados cooperem [Tsolkas et al. 2017]. Em uma direção baseada em pelotão considerando a teoria dos jogos, espera-se que cada veículo escolha sua estratégia sem ter acesso à estratégia dos outros veículos e sem aguardar a decisão dos mesmos, ou seja, um jogo não cooperativo e simultâneo.

O restante deste artigo é organizado da seguinte forma. A Seção 2 apresenta os objetivos e principais contribuições do trabalho. A Seção 3 apresenta os trabalhos relacionados. A Seção 4 descreve o cenário de avaliação e os resultados obtidos. Na Seção 5 são descritas as publicações relacionadas ao trabalho. A Seção 6 apresenta as conclusões.

\section{Objetivos e Principais Contribuições}

Levando em consideração os desafios e problemas levantamos, este artigo apresenta um protocolo baseado em teoria de jogos para a condução em pelotão (GT4P), o qual decide quais veículos devem participar do pelotão e servirão como um nó de retransmissão de vídeo tanto no cenário urbano quando no rodoviário. As principais contribuições da dissertação podem ser listadas como: (i) Um protocolo para selecionar os melhores nós de retransmissão, com base em informações de mobilidades; e (ii) Avaliar o comportamento 
do protocolo para transmissão de vídeo de acordo com as métricas de QoE em cenários urbanos e rodoviário. Assim, podemos resumir cada uma das contribuições:

Protocolo para selecionar os melhores nós de retransmissão, com base em informações de mobilidades. Conforme descrito no Capítulo 4 de [Lobato 2019], o estabelecimento de um pelotão começa assim que um veículo fonte recebe uma solicitação para enviar um vídeo para um veículo de destino. O GT4P seleciona os membros do pelotão com base em informação de direção, velocidade, percurso dos veículos, RSSI e distância dos vizinhos. Desta forma, o GT4P seleciona para participar do pelotão um conjunto de veículos que se movem na mesma direção com velocidades semelhantes e uma distância adequada para disseminar o vídeo, proporcionando maior conectividade entre os veículos. Como os veículos do pelotão alteram o tempo de viagem, o consumo de combustível e as emissões de $\mathrm{CO}_{2}$, a cooperação entre veículos é garantida por meio de uma função de recompensa que assegura uma retribuição aos participantes do pelotão de acordo com seus esforços no processo de transmissão de vídeo com suporte a QoE. Além disso, o GT4P ajusta a distância entre os membros do pelotão para reduzir os efeitos da comunicação sem fio na perda de pacotes. Desta forma, os membros do pelotão colaboram com a transmissão de vídeo, mitigando os efeitos da mobilidade do veículo e provendo disseminação de vídeo com suporte a QoE em cenários urbanos e rodoviários.

Avaliar o comportamento do protocolo para transmissão de vídeo de acordo com as métricas de QoE. A avaliação, descrita no Capítulo 5 de [Lobato 2019], foi realizada para analisar e examinar o desempenho do protocolo proposto para disseminar vídeos em dois cenários, urbano e rodoviário. Com base nos resultados obtidos, pode-se concluir que o GT4P é um protocolo eficiente para transmissão de vídeos com suporte a QoE tanto operando no cenário urbano quanto no rodoviário comparado a protocolos existentes, conforme pode ser visto na Seção 4 .

\section{Trabalhos Relacionados}

[Chen et al. 2011] considerou a teoria dos jogos para formar grupos e oferecer incentivos para os membros do grupo. Este trabalho pode ser usado para tomada de decisão em protocolos de roteamento, forçando a cooperação entre nós da rede. Ele considera o tamanho do armazenamento de cada veículo para decidir quais veículos participam da transmissão de dados. O esquema de incentivo fornece estímulo efetivo para os nós cooperarem e evita a degradação do desempenho do sistema nas VANETs com veículos egoístas. [Gerla et al. 2014 apresentou uma abordagem usando a teoria dos jogos para escolher os nós de encaminhamento para aplicar a codificação de rede, os autores também apresentaram tecnologias e protocolos para distribuição de conteúdo em VANETs.

[Amoroso et al. 2011] introduziu o Furthest Distance (FD), que considera um algoritmo de seleção de retransmissão de múltiplo salto orientada a remetente. No FD, antes de encaminhar um pacote, cada nó calcula uma Contention Window (CW) com base em sua localização atual e na localização dos vizinhos contidos no pacote recebido. Em geral, uma CW mais baixa significa que o veículo está mais próximo do destino, o que aumenta a probabilidade desse nó ser selecionado como um veículo pertencente ao pelotão. O objetivo do FD é minimizar o número de saltos entre a origem e o destino.

O BiDirectional Stable Communication (BDSC), proposto por [Rehman et al. 2016], considera que cada veículo transmite periodicamente uma mensagem HELLO, que contém três informações: ID do emissor, informações de localização e lista de nós de comunicação ativa (ACNL - Active Lista de nós de 
comunicação). Especificamente, o ID do emissor refere-se ao endereço MAC do nó de origem, enquanto as informações de localização referem-se às suas coordenadas de localização atuais. O ACNL é o componente mais importante dos pacotes HELLO, pois mantém as informações recentes dos veículos de salto único disponíveis, trocando constantemente os pacotes HELLO.

[Zhang and Cao 2011] propôs o V-PADA, que é uma solução de acesso a dados com reconhecimento de pelotão de veículos para VANETs. O V-PAD é um protocolo de pelotão que inclui dois componentes baseados em custo e eficiência para encontrar o melhor veículo para replicar dados no pelotão e também para analisar possíveis anomalias de mobilidade que podem afetar a transmissão de dados.

[Amoozadeh et al. 2015] introduziu um protocolo de gerenciamento de pelotão que considera uma abordagem centralizada de coordenação de pelotão, na qual o líder do pelotão coordena todas as comunicações. O protocolo considera três manobras básicas: mesclar, dividir e mudar de faixa com base nas VANETs e Cooperative Adaptive Cruise Control (CACC). Um conjunto de micro comandos trocados entre veículos que utilizam o IEEE 802.11p são usados para realizar as manobras básicas e a operação do protocolo é descrita em detalhes usando várias máquinas de estado finito.

[Jia et al. 2014] leva em consideração a mobilidade do veículo para estabelecer um pelotão, como fluxo de tráfego, largura de banda, velocidade e tamanho do pelotão. $\mathrm{O}$ autor considera a distribuição de probabilidade do fluxo de tráfego baseado em pelotão, incluindo espaçamento entre pelotões e espaçamento entre líderes de pelotão. Além disso, investigou a conectividade entre pelotões em um cenário prático de rodovia bidirecional e avaliou o tempo esperado de entrega de mensagens de segurança entre pelotões.

Com base na análise do trabalho relacionado, foi possível concluir que a disseminação de vídeo considerando pelotão atenua a influência do curto tempo de contato entre os veículos e evita falhas de comunicação, atrasos e perda de pacotes durante as transmissões de vídeo. Dessa maneira, os veículos que participam do pelotão podem encaminhar os pacotes de vídeo entre os veículos de origem e de destino dentro do pelotão, permitindo a transmissão de vídeo com menores taxas de desconexões. No entanto, o protocolo deve selecionar veículos adequados para participar do pelotão com base em informações de distância, velocidade, direção, qualidade do enlace e percurso. Isso envolve estar ciente dos requisitos de QoE e considerar mecanismos de incentivo para recompensar os veículos pela participação no pelotão.

\section{Avaliação e Resultados Obtidos}

Foram realizadas simulação usando o Veins, um framework do OMNeT ++, que implementa a pilha de protocolos IEEE 802.11p padrão para comunicação do veículo e um modelo de obstáculos para atenuação do sinal. Foi empregado o Simulador de Mobilidade Urbana (SUMO) para a simulação de tráfego e mobilidade de veículos. Isso permite reproduzir os movimentos desejados do veículo e interações V2V com base em dados empíricos. Foram realizadas simulações nos cenários urbano e rodoviário. Para o cenário da rodovia, foi considerado as rodovias do trace de Luxemburgo [Codeca et al. 2015], o qual abrange uma área de $156 \mathrm{~km}^{2}$ e $932 \mathrm{~km}$ de estradas para 26 horas de simulação de mobilidade para a cidade de Luxemburgo. Para o cenário urbano, foi utilizado o cenário Manhattan Grid, composto por dez ruas de pista dupla, espaçadas igualmente, em uma área de $1 \mathrm{~km}^{2}$. Foram transmitidos sequências de vídeo com diferentes níveis de movimentação e complexidade. Esses vídeos têm duração entre 10 e 20 segundos, 
codificados com um codec H.264 a 300 kbps. As simulações são executadas por 1000 segundos, onde o veículo fonte envia o vídeo a qualquer momento após os 100 segundos iniciais e antes dos últimos 100 segundos. Também foram considerados os efeitos de atenuação de sinal causados por edifícios. Para quantificar a evolução do tráfego nesse cenário, definiu-se a densidade de veículos entre 200 e 400 veículos $/ \mathrm{km}$.

A Figura 1 mostra o QoE dos vídeos entregues pelos protocolos FD, BDSC, P2V e GT4P em um cenário urbano com diferentes densidades por meio das métricas de SSIM e VQM. Analisando os resultados da Figura 1(a), concluí-se que o SSIM dos vídeos fornecidos pelo protocolo GT4P aumenta assim que a densidade do veículo aumenta, pois o cenário possui mais veículos candidatos para ingressar no pelotão, melhorando a decisão. Além disso, o protocolo GT4P entregou vídeos com SSIM 6\%, 54\% e 44\% superior em comparação com P2V, BDSC e FD, respectivamente. Isso ocorre porque o GT4P estabeleceu um pelotão selecionando veículos com velocidade semelhante, distância apropriada entre os membros do pelotão, trajetória de viagem semelhante e boa qualidade do enlace, assim como o GT4P ajusta a distância entre os membros do pelotão para evitar desconexões. O pelotão fornece uma comunicação V2V confiável durante a transmissão de vídeo, mitigando os efeitos da mobilidade do veículo para evitar falhas de comunicação, atrasos, área vazia e perda de pacotes.

A Figura 1(b) mostra o VQM para vídeos entregues via protocolos FD, BDSC, P2V e GT4P em um cenário urbano. Ao contrário dos valores do SSIM, os baixos valores de VQM significam um nível de qualidade de vídeo mais alto. Os resultados do VQM confirmam os benefícios do GT4P para transmitir vídeos com suporte a QoE, estabelecendo um pelotão para evitar os efeitos da mobilidade do veículo na QoE. Tendo como exemplo, o GT4P transmitiu pacotes de vídeo com uma taxa de perda de quadros reduzida, protegendo os quadros prioritários em períodos de congestionamento e erro de enlace [Greengrass et al. 2009]. O GT4P reduziu a taxa de perda de quadros em 40\%, $44 \%$ e $4 \%$ em comparação com a transmissão de vídeo via FD, BDSC e P2V, respectivamente, conforme pode ser visto com mais detalhes no Capítulo 5 de [Lobato 2019].

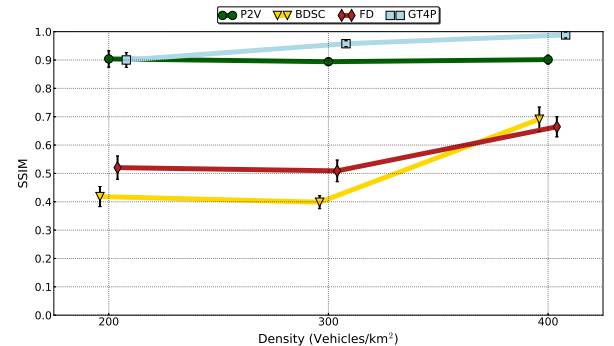

(a) SSIM por densidade

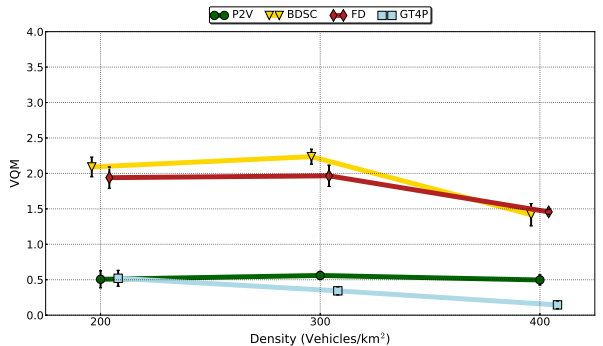

(b) VQM por densidade

Figura 1. QoE para vídeos transmitidos em um cenário urbano

A Figura 2 mostra o SSIM e VQM para vídeos com diferentes níveis de movimento e complexidade transmitidos via protocolos FD, BDSC, P2V e GT4P em um cenário de rodovia. Analisando os resultados da Figura 2(a), concluí-se que o GT4P entregou vídeos com alto SSIM em comparação com FD, BDSC e P2V, independentemente dos níveis de movimento e complexidade do vídeo. Por exemplo, os vídeos entregues pelo GT4P têm valores de SSIM mais próximos de 1. Por outro lado, os vídeos entregues por P2V, BDSC e FD reduziram o SSIM em $11 \%, 60 \%$ e $48 \%$ em comparação 
ao GT4P, respectivamente. Isso ocorre porque o GT4P estabeleceu um pelotão selecionando veículos com velocidades semelhantes, distância apropriada entre os membros do pelotão, trajetória de viagem semelhante e boa qualidade do enlace. Esses resultados confirmam que o GT4P também fornece vídeo com bom nível de qualidade em um cenário de rodovia. Os diferentes valores de avaliação de vídeo são devidos às características únicas de cada sequência de vídeo, onde pequenas diferenças de movimento e nível de complexidade podem influenciar os valores obtidos [Immich et al. 2013]. Dessa forma, é importante realizar os experimentos em vídeos com diferentes características. A Figura 2(b) mostra a QoE medido por meio do VQM para vídeos com diferentes níveis de movimento e complexidade transmitidos pelos protocolos FD, BDSC, P2V e GT4P em um cenário VANET de rodovia. Mais uma vez, os resultados do VQM confirmam os benefícios do GT4P para transmitir vídeos com suporte a QoE, estabelecendo um pelotão para evitar os efeitos da mobilidade do veículo na qualidade do vídeo.

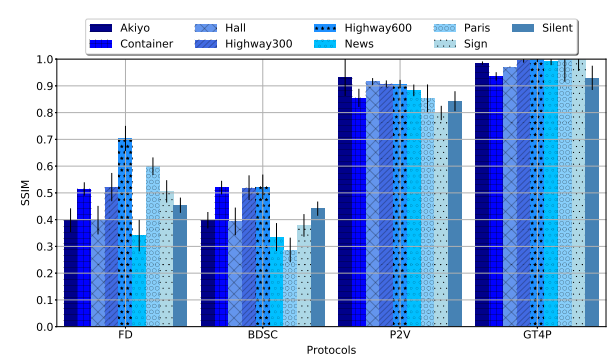

(a) SSIM por vídeo

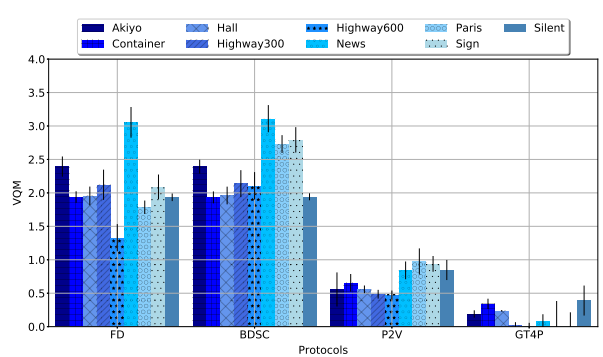

(b) VQM por vídeo

Figura 2. QoE para vídeos transmitidos em um cenário de rodovia

Com base nos resultados obtidos, pode-se concluir que o GT4P é um protocolo eficiente para transmissão de vídeos tanto operando no cenário urbano quanto no rodoviário. Ademais, avaliações adicionais de atraso por vídeo e o comportamento dos frames de vídeo podem ser consultados na Subseção 5.2 e 5.3 do Capítulo 5 de [Lobato 2019].

\section{Publicações}

Como resultado desta dissertação de mestrado, foram publicados cinco artigos em anais de conferências nacionais e internacionais de prestígio e grande impacto na área de redes e sistemas distribuídos, onde um dos trabalhos foi premiado como melhor artigo. Além disso, um artigo foi aceito em um periódico internacional. O impacto do trabalho pode ser medido por meio do número de citações do Google acadêmico, onde os artigos publicados receberam um total de 25 citações, resultado em um índice h de 2 e um índice i10 de 2. Assim, segue a lista em detalhes dos artigos aceitos

- 2018 - Wireless Communications and Mobile Computing. [Qualis A2, CiteScore 1.470, 10 citações]

- 2017 - IEEE Global Communications Conference (GLOBECOM). [Qualis A1, 13 citações]

- 2017 - Workshop em Desempenho de Sistemas Computacionais e de Comunicação (WPERFORMACE). [Qualis B3]

- 2018 - IEEE International Conference on Communications Workshops (ICC Workshops): 5G and Cooperative Autonomous Driving. [3 citações]

- 2018 - Workshop de Gerência e Operação de Redes e Serviços (WGRS).

- 2018 - IEEE Latin-American Conference on Communications (LATINCOM). Best Paper Award 


\section{Conclusão}

Este artigo resume as contribuições da dissertação de mestrado em [Lobato 2019]. A transmissão de vídeo em VANETs com suporte à QoE é uma tarefa desafiadora devido às características específicas das VANETs, como a constante mudança na estrutura topológica da rede, causada pelo curto tempo de contato entre os veículos e a mobilidade altamente dinâmica. Para solucionar esses desafios, foi apresentado o protocolo GT4P com o intuito de formar pelotões para disseminação de vídeo entre os veículos. O GT4P atenua os problemas relacionados a desconexões frequentes causadas pela alta mobilidade do veículo, estabelecendo um pelotão com base na direção, velocidade, distância, RSSI e trajetória dos veículos. Além disso, o protocolo GT4P impõe que os veículos do pelotão mantenham uma determinada distância para mitigar falhas de rota e perda de pacotes. O protocolo GT4P considera um jogo não cooperativo e simultâneo, em que o comportamento cooperativo é imposto por meio de uma função recompensa para incentivar os veículos a ingressarem no pelotão. Dessa forma, o GT4P aumenta a conectividade entre os veículos, reduzindo a perda de pacotes durante a transmissão de vídeo.

Simulações foram executadas para a análise do comportamento do protocolo para disseminação de vídeo em dois cenários diferentes: Urbano e Rodoviário. Com base em resultados de simulação foi identificado que o protocolo GT4P entregou vídeos com QoE $10 \%, 60 \%$ e $50 \%$ superiores aos vídeos entregues pelos protocolos P2V, BDSC e FD, respectivamente. Isso ocorre porque o GT4P estabelece um pelotão levando em consideração a direção, a velocidade, a distância, a qualidade do enlace e o percurso do veículo, o que reduz o impacto da mobilidade do veículo na transmissão de vídeo. Portanto, os resultados da simulação mostram a eficiência do GT4P em comparação com os protocolos P2V, BDSC e FD para garantir a disseminação de vídeo com QoE satisfatória em cenários rodoviários e urbanos.

\section{Referências}

[Ahmad et al. 2019] Ahmad, I., Noor, R. M., Zaba, M. R., Qureshi, M. A., Imran, M., and Shoaib, M. (2019). A cooperative heterogeneous vehicular clustering mechanism for road traffic management. International Journal of Parallel Programming, pages 1-20.

[Amoozadeh et al. 2015] Amoozadeh, M., Deng, H., Chuah, C.-N., Zhang, H. M., and Ghosal, D. (2015). Platoon Management with Cooperative Adaptive Cruise Control Enabled by VANET. Vehicular Communications, 2(2):110-123.

[Amoroso et al. 2011] Amoroso, A., Marfia, G., and Roccetti, M. (2011). Going Realistic and Optimal: A Distributed Multi-Hop Broadcast Algorithm for Vehicular Safety. Computer Networks, 55(10):2504-2519.

[Chen et al. 2011] Chen, T., Wu, L., Wu, F., and Zhong, S. (2011). Stimulating Cooperation in Vehicular Ad Hoc Networks: a Coalitional Game Theoretic Approach. IEEE Transactions on Vehicular Technology, 60(2):566-579.

[Codeca et al. 2015] Codeca, L., Frank, R., and Engel, T. (2015). Luxembourg sumo traffic (lust) scenario: 24 hours of mobility for vehicular networking research. In Proceedings of the IEEE Vehicular Networking Conference (VNC), pages 1-8.

[Gerla et al. 2014] Gerla, M., Wu, C., Pau, G., and Zhu, X. (2014). Content Distribution in VANETs. Vehicular Communications, 1(1):3-12. 
[Greengrass et al. 2009] Greengrass, J., Evans, J., and Begen, A. C. (2009). Not All Packets are Equal, Part 2: The Impact of Network Packet Loss on Video Quality. IEEE Internet Computing, 13(2):74-82.

[Husain et al. 2019] Husain, K., Awang, A., Kamel, N., and Aïssa, S. (2019). Intersectionbased link-adaptive beaconless forwarding in urban vehicular ad-hoc networks. Sensors, 19(5):1242.

[Immich et al. 2013] Immich, R., Cerqueira, E., and Curado, M. (2013). Adaptive Videoaware FEC-based Mechanism with Unequal Error Protection Scheme. In Proceedings of the 28th annual ACM symposium on applied computing (SAC), pages 981-988.

[Jia et al. 2014] Jia, D., Lu, K., and Wang, J. (2014). On the network connectivity of platoon-based vehicular cyber-physical systems. Transportation Research Part C: Emerging Technologies, 40:215-230.

[Li et al. 2018] Li, Y., Tang, C., Li, K., He, X., Peeta, S., and Wang, Y. (2018). Consensusbased cooperative control for multi-platoon under the connected vehicles environment. IEEE Transactions on Intelligent Transportation Systems, 20(6):2220-2229.

[Liu et al. 2016] Liu, J., Wan, J., Wang, Q., Deng, P., Zhou, K., and Qiao, Y. (2016). A Survey on Position-based Routing for Vehicular Ad Hoc Networks. Telecommunication Systems, 62(1):15-30.

[Lobato 2019] Lobato, W. (2019). Platoon-based driving protocol for multimedia transmission over vanet. Master's thesis, Universidade Federal do Pará.

[Lopez-Martin et al. 2018] Lopez-Martin, M., Carro, B., Lloret, J., Egea, S., and SanchezEsguevillas, A. (2018). Deep learning model for multimedia quality of experience prediction based on network flow packets. IEEE Comm. Magazine, 56(9):110-117.

[Rehman et al. 2016] Rehman, O., Ould-Khaoua, M., and Bourdoucen, H. (2016). An adaptive relay nodes selection scheme for multi-hop broadcast in vanets. Computer Communications, 87:76-90.

[Tsolkas et al. 2017] Tsolkas, D., Liotou, E., Passas, N., and Merakos, L. (2017). A survey on parametric qoe estimation for popular services. Journal of network and computer applications, 77:1-17.

[Ulin 2019] Ulin, J. C. (2019). The business of media distribution: Monetizing film, TV, and video content in an online world. Routledge.

[Uppoor and Fiore 2012] Uppoor, S. and Fiore, M. (2012). Insights on Metropolitan-scale Vehicular Mobility from a Networking Perspective. In Proceedings of the 4th ACM international workshop on Hot topics in planet-scale measurement, pages 39-44. ACM.

[Zhang and Cao 2011] Zhang, Y. and Cao, G. (2011). V-PADA: Vehicle-Platoon-Aware Data Access in VANETs. IEEE Trans. on Vehicular Technology, 60(5):2326-2339. 